\title{
A Data Acquisition and Processing Method for Logistics AGV Obstacle Detection LiDAR
}

\author{
Tianxiang Yuan ${ }^{\mathrm{a}}$, Jie Zhu ${ }^{\mathrm{b}, *}$ and Huizhi Gao ${ }^{\mathrm{c}}$ \\ School of Information, Beijing Wuzi University, Beijing, China \\ a253979496@qq.com, bzhujie@bwu.edu.cn, c1543931886@qq.com \\ * Tian-Xiang Yuan
}

Keywords: Automated Guided Vehicle, Avoid obstacles, LiDAR, The data collection, The data processing.

\begin{abstract}
In the semi-structured logistics warehouse AGV (Automated Guided Vehicle) to achieve environmental awareness is the key to its own obstacle avoidance. In order to realize the purpose of logistics AGV, the paper first analyzes the working principle of LiDAR, and then designs and realizes the data acquisition and control of LiDAR. Finally, it is applied to the laser radar data processing is performed, including the reduction of the number of invalid data points and the adaptive threshold of the nearest neighbor clustering for regional segmentation. The results show that the laser radar data acquisition system and the real-time and effect of clustering segmentation have good effect on the subsequent feature point extraction and obstacle judgment.
\end{abstract}

\section{Preface}

AGV (Automated Guided Vehicle) is equipped with electromagnetic or optical and other automatic guide device, it can be without manual guidance in the case of pre-set a good route to automatically guide to the destination, with security and a variety of Transfer function of the logistics transport car. You can achieve the loading and unloading of goods, handling the entire process of all automation. Intelligent, automated AGV is brought about by the efficiency of the increase, so in the storage industry, manufacturing, tobacco industry, electric business warehouse has a wide range of applications ${ }^{[1]}$.

AGV is currently able to travel at $2 \mathrm{~m} / \mathrm{s}$ in unknown semi-structured warehouses, manufacturing plants or port environments. In order to protect the environment in the process of equipment, personnel and car their own safety, AGV security system is particularly important. Safety system consists of high-precision sensor and contact emergency stop collision device two levels of security protection, generally before and after the assembly. Applied to the logistics AGV barrier detection methods are generally based on stereoscopic vision, laser radar, color cameras and other obstacles detection ${ }^{[2]}$.

AGV safety module in the laser radar data acquisition and processing has always been the key to the study, the current laser radar data acquisition and processing research is divided into two categories, one is focused on an application area or industry, that is, dedicated collection and processing System; Second, the general collection and processing system ${ }^{[3]}$. Dedicated system generally for a particular industry, relatively poor system reuse and the development of difficult. General system is not the only standard, and hardware software is not open, increasing the difficulty of secondary development. This paper first analyzes the working principle of the laser radar, and then designs and realizes the data acquisition and control of the lidar in the AGV safety system. Finally, the laser radar is used for the AGV anti-collision function. The nearest neighbor clustering of the adaptive threshold is divided into regions, which provides the basis for the subsequent development of obstacle detection.

\section{The Working Principle of the LiDAR}

Before studying the laser radar data acquisition and processing, we must first understand the working principle of the lidar. LiDAR used in logistics AGV anti-collision function is mainly through 
the launch of laser pulses into the environment measured by the physical surface, part of the light will be reflected by the laser radar receiver to receive, according to the equipment equipped with the timer recording time, Is known, you can find the distance from the logistics AGV, AGV through the control unit to avoid the measured object, so as to achieve the purpose of avoiding obstacles ${ }^{[4]}$. The laser ranging formula (1) is as follows:

$\mathrm{D}=1 / 2 \mathrm{VT}$.

Where $\mathrm{D}$ is the distance between the lidar and the measured object, $\mathrm{V}$ is the speed of light, and $\mathrm{T}$ is the time interval at which the laser pulse is emitted to the received time.

The detection of obstructions by laser radar can obtain the surrounding environment information in real time. Because of its high operating frequency, high precision of measurement data and low cost, most of the safety units in AGV adopt laser radar detection technology. The working mode and scanning direction of the laser radar on the logistics AGV are as follows:

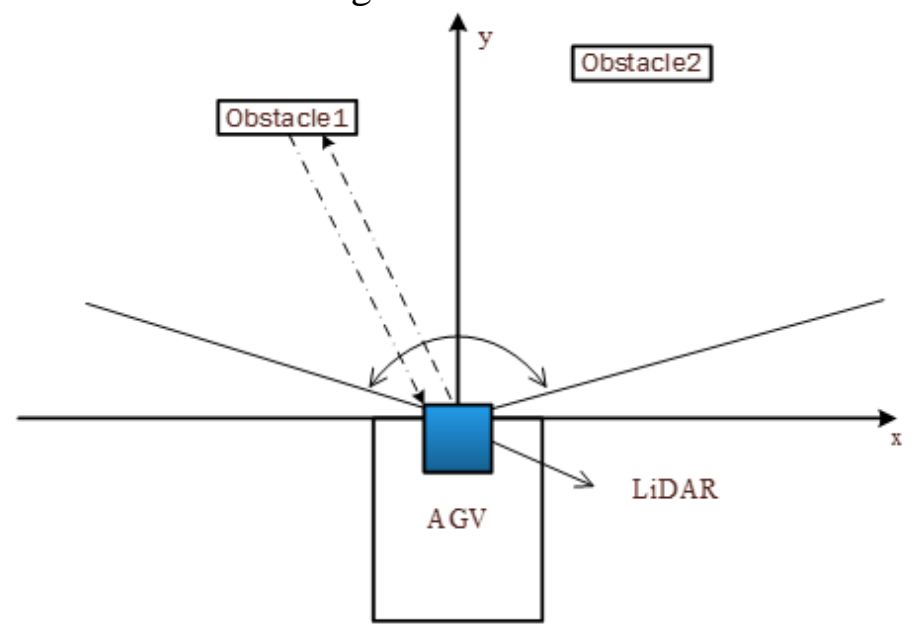

Figure 1 Logistics AGV obstacle avoidance LiDAR scanning direction

\section{Data Collection and Processing of LiDAR}

\subsection{LiDAR Data Collection}

This paper uses the domestic low-cost LiDAR LS01C, scanning angle resolution of 1 degree, scanning interval of 360 degrees, scanning distance of 6 meters range, summed up the single-line laser radar small size, light weight, fast frame width, Measurement accuracy is relatively high, etc., for the logistics AGV for the detection of obstacles. Figure 2 shows the LS01C single frame scan data.

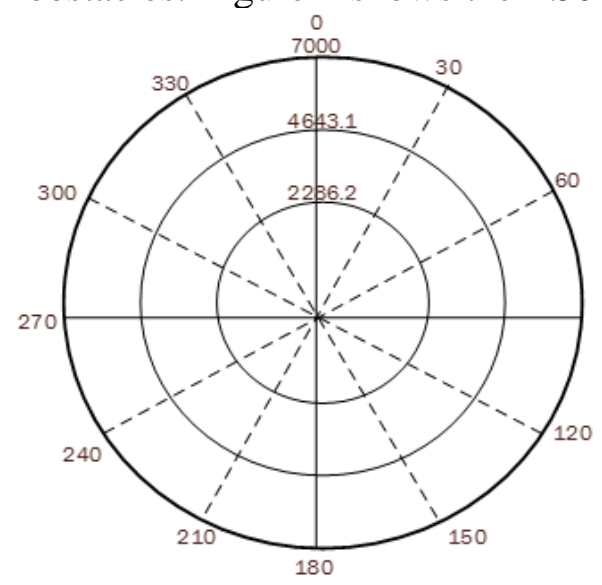

Figure 2 LS01 single-frame scan data diagram

In the LiDAR data acquisition system, the software is the most intuitive form of its collected data. In order to better collect the hardware system data, the system uses the Microsoft Foundation Classes (Microsoft Foundation Classes) Microsoft Foundation Class Library to design the laser radar data 
acquisition visualization interface to facilitate the experimental stage to understand the logistics AGV front environmental information, Data processing, but also the collection of data were stored.

The data acquisition system mainly through the link CSerialPort class, configure the laser radar communication parameters, through the instructions provided by the manufacturers to obtain the laser radar collection of environmental data, the collected environmental data after the analysis through the link in the Microsoft Foundation class CListCtrl control The environment data is displayed in two columns of data tables. The CListCtrl class encapsulates the "list view control" function, not only for the displayed icons, but also for each tab to collect, with good display data. At the same time, the need to collect the data collected by the laser radar into a certain data format, by reference \#include iostream.h, \# include fstream.h header file, the memory of the data stream into the file to save to the definition of storage space. LIDAR data collected by the angle data and distance data 2 sets of data, save two columns to distinguish the angle and distance, post-processing analysis. After several experiments, the data acquisition system can be very good real-time data collection without interruption.

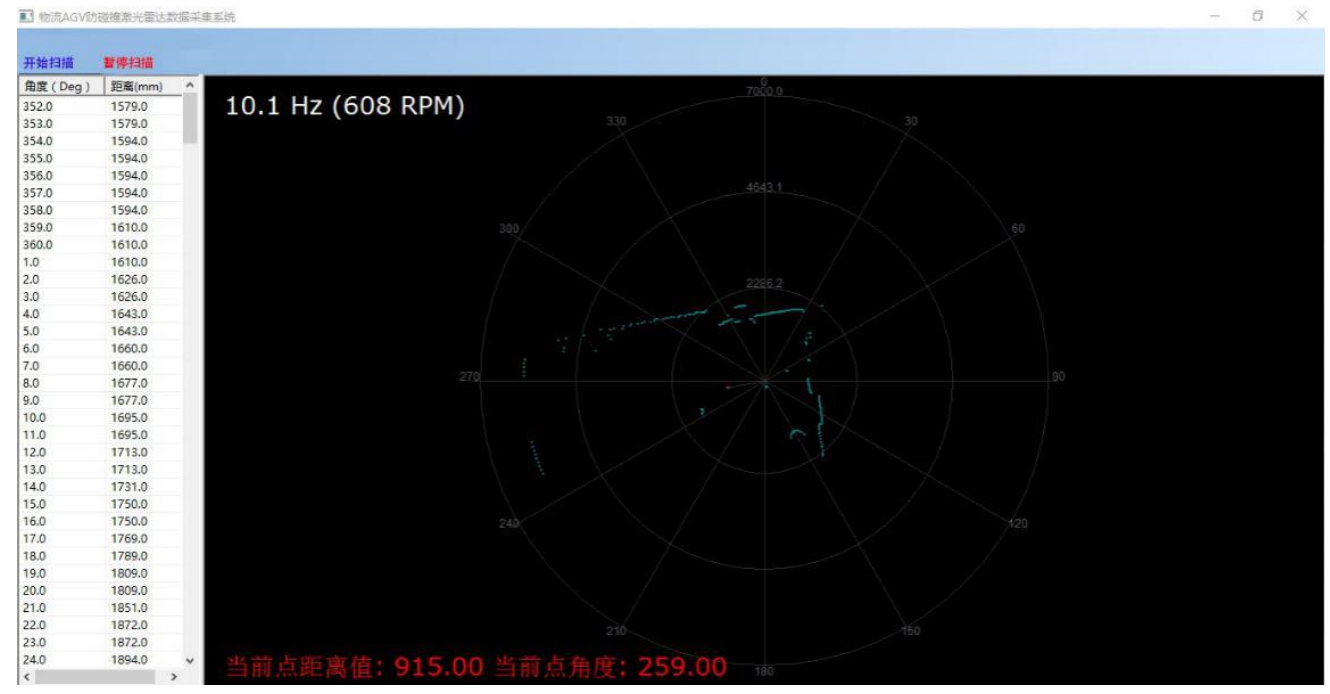

Figure 3 LS01 data acquisition interface

\subsection{Data Processing of LiDAR}

Data processing is the processing and transformation of data collected by LIDAR, finding valid data in a large number of complex data, and analyzing the useful and needed results by means of scientific methods ${ }^{[5-6]}$.

\subsubsection{Filter out Noise Points}

LiDAR placed on the logistics AGV in the storage or manufacturing workshop environment, the data will inevitably be affected by the surrounding environment, the surrounding obstacles of the material reflectivity and the environment of dust and so will affect the laser radar for the surrounding environment perception effect. The noise generated by the LiADR will interfere with the detection of the target obstacle, that is, the clustering process, and will eventually make an erroneous judgment on whether the logistics AGV avoids the obstacle and cause unnecessary economic losses.

(1) Remove the invalid range of points

Due to the limitations of the LS01C measurement range, the points outside the range or outside the effective range are measured under the interference factor. These data points are unreliable or the data points that we do not need to analyze. Give away. To sum up, in order to retain valid data points, given a limited range, we only to the effective range of data points to do the processing, and the effective range of data points can be called irrelevant data points, not for processing analysis The LS-01C measuring range of 6 meters, select the effective range of 5 meters. The method of judging the data points outside the range is as follows(2): 
scanPoint.value $=\left\{\begin{array}{l}\text { True(in range) } \\ \text { False(out of range) }\end{array}\right.$

(2) Processing of isolated data points

For the effective measurement range of the lidar, there will be some isolated data points, or there are fewer areas of data points. Isolated data points are generally two forms of expression:

1)Zero distance point, this is because the lidar should have detected the echo where the echo was not detected, The general method of judging is to see whether there is a similar distance value between the data points before and after the zero distance point, and it is judged that the point is an isolated point. The specific method is assigned to the zero distance point by the average of the distance values of the data points before and after the isolated point, and the isolated point is filled, and the global data value is accurate. In other cases, the zero value is kept constant. (3) is

expressed as:

$$
\operatorname{Dis}(\mathrm{i})=\left\{\begin{array}{lc}
(\operatorname{Dis}(\mathrm{i}-1)+\operatorname{Dis}(\mathrm{i}+1)) / 2 & (\operatorname{Dis}(\mathrm{i}-1), \operatorname{Dis}(\mathrm{i}+1)) \neq 0 \\
0 & \text { Otherwise }
\end{array}\right.
$$

2)Non-continuous isolated points, LIDAR scan point cloud data, there are some less data points Region, in order to eliminate isolated points away from the continuous point, using the median filter method. The median filter is based on the ranking statistics theory. The basic principle is that the discrete data sequence of the laser radar acquisition data points is $\{X(i) \mid i=1,2, \ldots, n\}$, and the kth position in the set data As the center, before and after a total of the same value, recorded as $\mathrm{m}, \mathrm{m}$ is generally the base, the $\mathrm{m}$ data in ascending or descending order of the way, the sorted intermediate value as the original sequence of the k-position value The $\mathrm{K}$ values in turn from small to large, to achieve the entire lidar data filtering. Median filtering can completely eliminate spikes and isolated noise points, but also to maintain the original data. Data primitive to ensure its accuracy, there will not be excessive treatment of data ${ }^{[7]}$. Figure 3 is to remove the noise point before and after the contrast effect map.
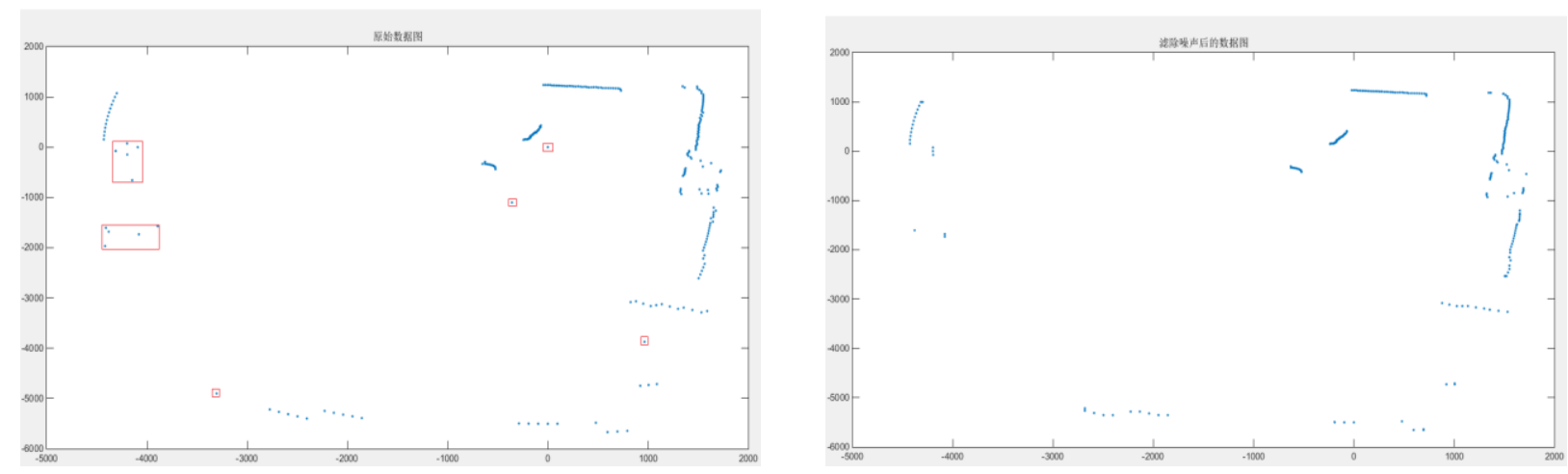

Figure 4 To remove the noise before and after the comparison effect map

\subsubsection{Minimal neighbor clustering}

In this paper, the logistics AGV platform is equipped with LS01C a single-line laser radar, single-line laser radar data obtained by each frame less, the distribution of the characteristics are more obvious. The most important clustering algorithm is clustering effect and algorithm time complexity, which is applied to logistics AGV. In order to avoid the surrounding obstacles in real time, the efficiency of clustering algorithm is very high. The general clustering algorithms for data processing include K-means clustering, density DBSCAN clustering, fuzzy clustering, and nearest neighbor clustering algorithm ${ }^{[8]}$. Aiming at the real - time and accuracy requirements of the AGV for obstacle detection and the data characteristics of the angular scanning of the lidar, the nearest neighbor clustering algorithm with adaptive threshold is proposed.

According to the scanning characteristics of the lidar, each frame of data collected, the echo returned on the same object appears as a series of consecutive data points, the distance is generally similar, if the two-point data distance mutation, reflected Is the echo of two different objects in the 
region. So the distance between the two adjacent points, the two points may belong to a class, two adjacent distance away, then the two data points do not belong to the same class may be relatively large. It is necessary to set up a standard to determine whether the distance value is too large or too small. The nearest neighbor clustering algorithm starts from the starting point of the scanning, starting from the second point, starting from the second point, calculating the Euclidean distance of the adjacent two points in turn, and then comparing the European distance between adjacent two points to the threshold, The distance between the two adjacent points is greater than the threshold, then the two points do not belong to the same class, between the two points of the European distance is less than the threshold, then the two belong to the same category.

The specific algorithm flow is shown in Figure 5 below:

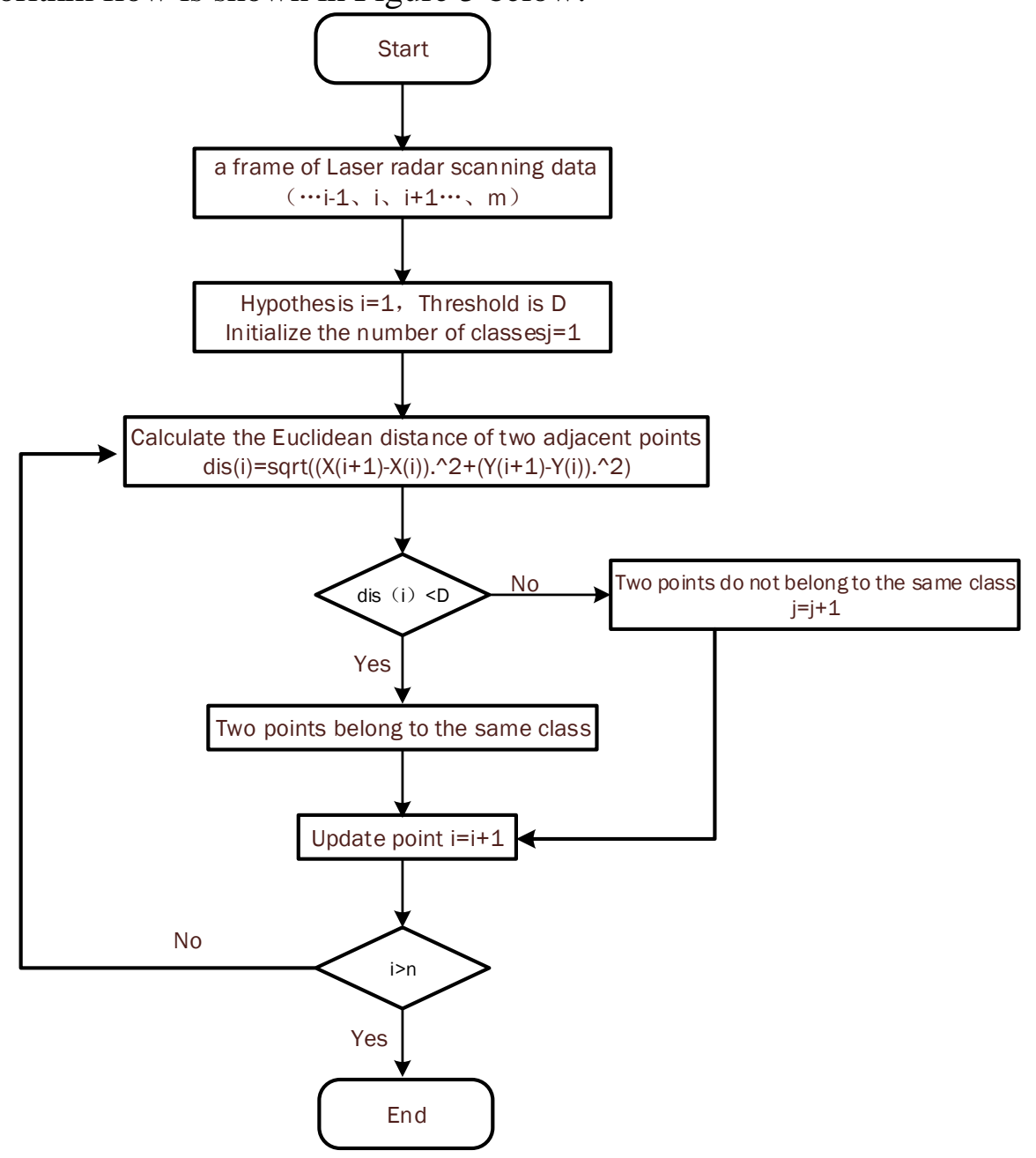

Figure 5 Near neighbor clustering algorithm flow chart

Among them, the nearest neighbor clustering algorithm to determine the threshold of the most critical. When the threshold is too small, too many types of clustering, regional segmentation confusion, when the threshold selection is too large, too many types of clustering, multiple obstacles may be clustered into a class, affecting the logistics AGV traffic efficiency.

As the laser beam of the laser radar has a fan-shaped scanning characteristic, the threshold value of the adjacent two laser reflection points increases correspondingly, and the distance between the adjacent two laser reflection points is linear with the extension of the beam Added as shown in Figure 6:

The formula is (4),(5):

$\mathrm{dr} \approx \mathrm{r}^{*} \sin (\mathrm{d} \theta)$

threshold $=a^{*} d r$.

In the above formula, $d$ is the angular resolution of the lidar, $r$ is the length of the laser, that is, the laser data point distance from the laser radar distance data, $\mathrm{dr}$ is the distance between two adjacent data 
points, a is the threshold coefficient, threshold is the threshold. The nearest neighbor clustering algorithm threshold is adaptive with the distance of each laser point, where the threshold coefficient a is desirable

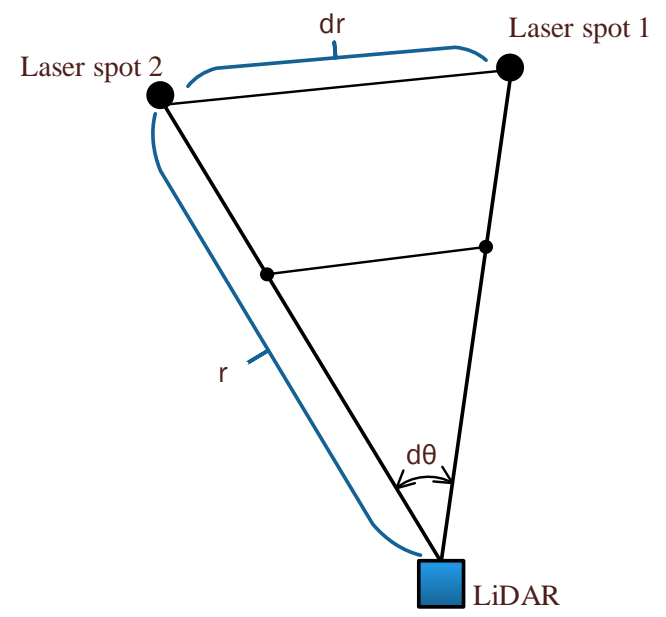

$5,10,50,100$

Figure 6 Distance between two laser reflection points

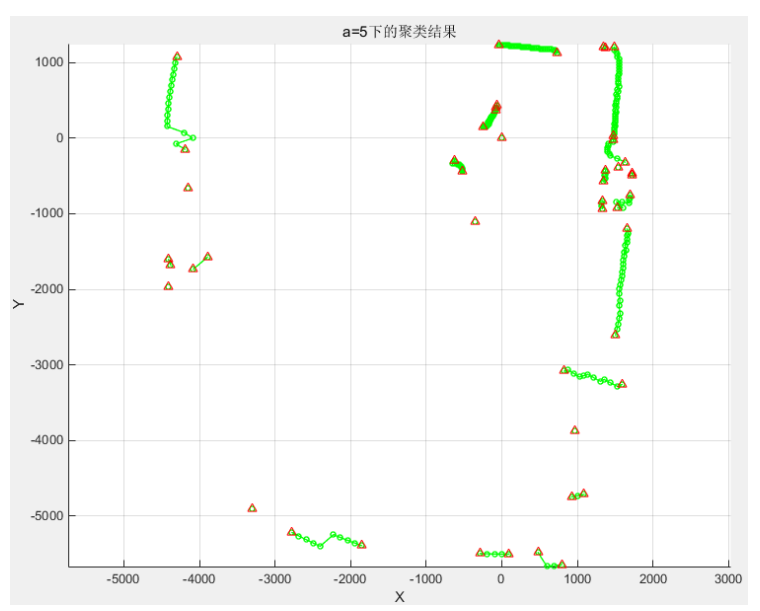

$\mathrm{a}=5$

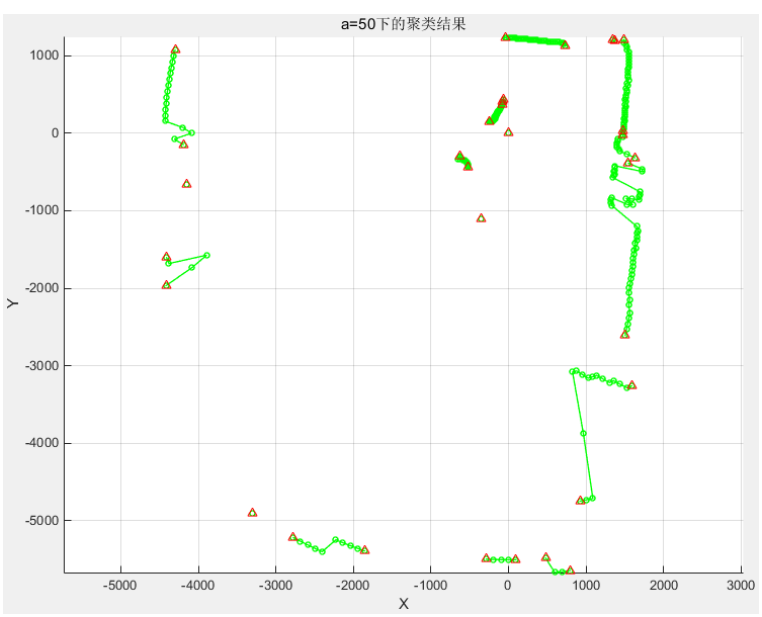

$a=50$

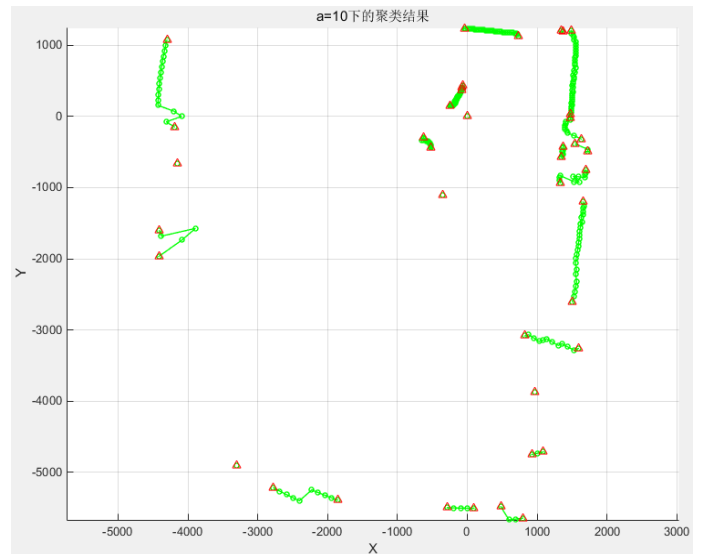

$\mathrm{a}=10$

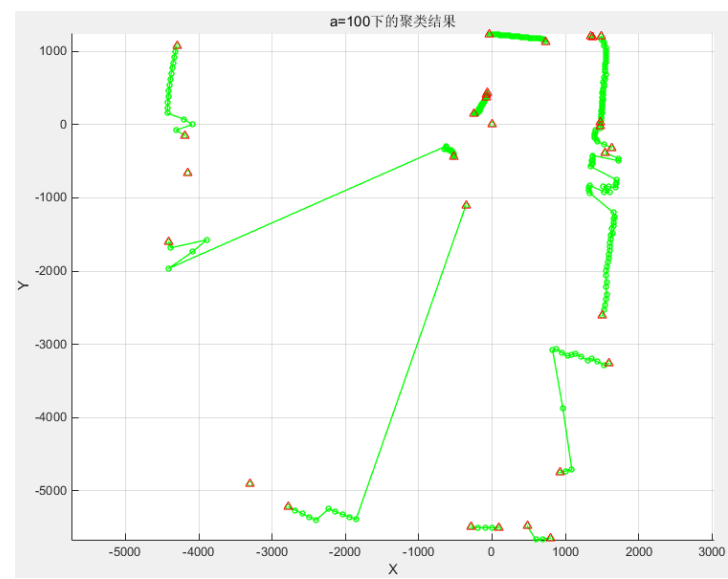

$\mathrm{a}=100$

Figure 7 clustering effect map with different threshold coefficients

The above clustering results are clustering results under different threshold coefficients. The red triangles appear in pairs, and the data points enclosed by a pair of red triangles are a kind of data points. Where the threshold coefficient is too small there will be too many types of clustering, post-logistics AGV identification of obstruction caused by a certain degree of miscarriage of justice, the threshold is too large coefficient, the adjacent obstruction after clustering into a class of obstacles, for small 
Obstacles, logistics AGV may cause missed calls, resulting in economic losses and the occurrence of security incidents. Compared with the above four different threshold coefficients clustering the results obtained, which select the results of $\mathrm{a}=10$ clustering is more appropriate.

Through the Nearest Neighbor Clustering algorithm, a detailed regional segmentation of the environmental scanning around the logistics AGV is carried out, which provides a better effect for the post-feature point extraction and line fitting. The purpose is to make the AGV better understand whether the surroundings exist Obstacles, in order to take obstacles in the event of better control measures to avoid the occurrence of economic losses and security incidents.

\section{Experimental results}

The result of clustering is to make a precise regional segmentation of a particular individual or object in each region of the actual environment in order to extract the levy points in the region after division. Figure 8 is the actual experimental environment and laser radar scan after the data processed by the plan. Which in the data on the right side of the concave area is the field environment in the door, the distant data points sparse mainly because the lidar detection range of 6 meters and the window glass reflex echo, so there is a breakpoint.

Different threshold coefficients have a certain impact on the clustering category, too many categories, too few to accurately identify the impact of obstacles. In this paper, we compare the clustering effect of the threshold coefficient $\mathrm{a}=10$ with the experimental environment, and the clustering results basically accord with the distinction and recognition of the individual or the object in the actual environment. Experimental environment and post-processing data shown in Figure 8:
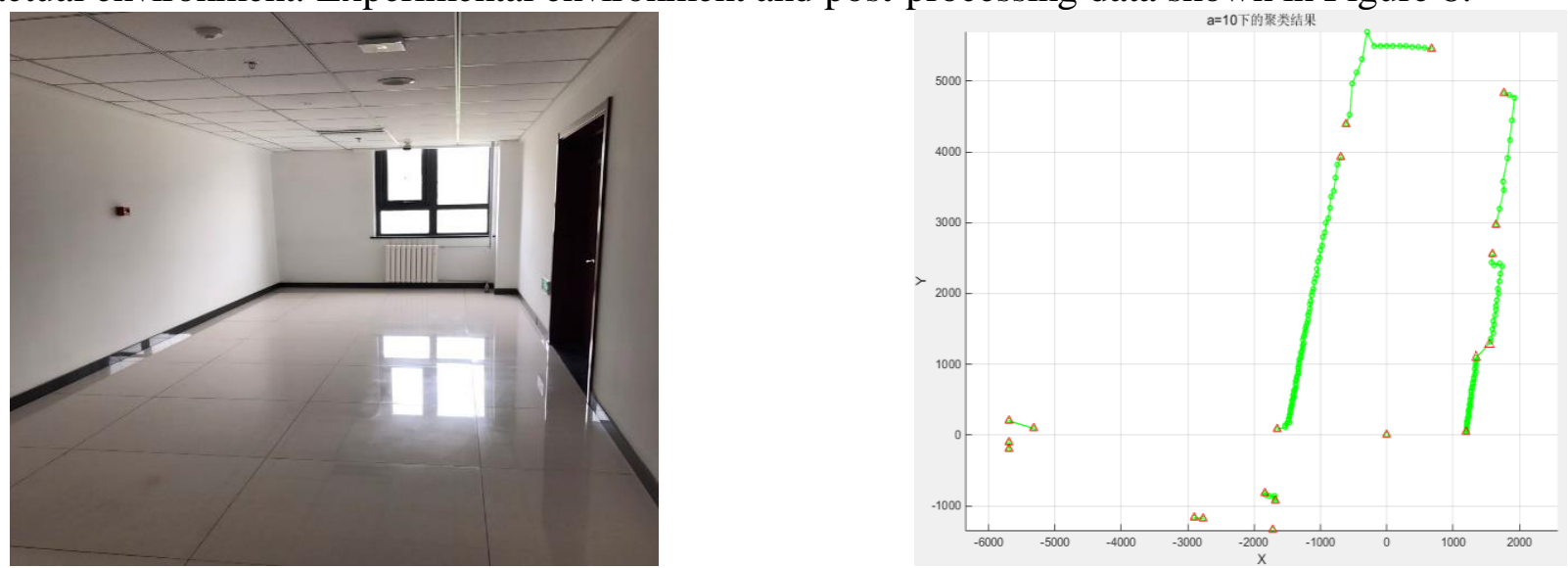

Figure 8 Comparison of experimental environment and post-clustering area segmentation

\section{Conclusion}

Logistics AGV security system requires laser radar, laser radar with high resolution, wide viewing angle, high efficiency, in the logistics of automatic guided car has a wide range of applications, not only used in obstacle avoidance function, but also used in map construction and positioning function , For the purpose of better path planning ${ }^{[9-10]}$. LiDAR scanning is AGV non-contact safety protection device performance of the most and the most secure security program, so for the logistics AGV Obstacle avoidance LiDAR data acquisition and data processing for the late whether the static obstacle or dynamic obstacles Recognition is of great importance. In this paper, the low-cost LiDAR in the application of AGV anti-collision function, to achieve visual interface real-time acquisition of logistics AGV around the environmental data and the data collected for noise reduction and nearest neighbor clustering for the late logistics AGV an accurate regional segmentation is identified for the surrounding obstacle identification. 


\section{Acknowledgements}

This paper is supported by the Funding Project for Technology Key Project of Municipal Education Commission of Beijing (ID:TSJHG201310037036); Funding Project for Beijing key laboratory of intelligent logistics system; Funding Project of Construction of Innovative Teams and Teacher Career Development for Universities and Colleges Under Beijing Municipality (ID:IDHT20130517), and Beijing Municipal Science and Technology Project (ID:Z131100005413004);Funding Project for Beijing philosophy and social science research base specially commissioned project planning (ID:13JDJGD013);Funding Project for Beijing Intelligent Logistics System Collaborative Innovation Center.

\section{References}

[1] Zhang Chen Besi, Huang Zhiqiu. Overview of the development of automatic navigation vehicle (AGV) [J]. China Manufacturing Information, 2010,01.

[2] Liu Zhiping, Zhai Junjie, Dong Xichen.GAGV Laser Scanning Crash Safety System [J]. Lifting and Transportation, 2006 (7): 42-44.

[3] Dai Yongjiang. Lidar technology [M]. Beijing: Electronic Industry Press, 2010.

[4] Wan Yao, Yao Juan, Li Weina, Li Lifang. Laser radar signal acquisition and processing research [J]. Sensor World, 2012: 27-34.

[5] LU Fen, OU Yiwen. Lidar radar data collection and processing research [J]. Laser magazine, 2016, 37 (9): 87-90.

[6] Zhan Qingping, Liang Yubin. Ltdra Data Processing, Information Extraction and Application [J] .Geographical Information World, 2011,4 (2): 38-39

[7] Shao Min, He Zhanxiang, Zhang Lien. Application of two-dimensional median filtering in electromagnetic noise reduction curve [J]. Journal of Geophysical and Geochemical Exploration, 2001, 23 (3): 227-232.

[8] Wu Weiyi, Liu University, Dai Bin. A clustering analysis method for processing lidar data [J]. Computer Simulation, 2007,24 (8): 236-240.

[9] Cui Lili, Wan Zhai. Safety and automation simultaneously - laser photoelectric sensor in the AGV application [J]. Modern Manufacturing, 2005,29: 62-64.

[10] Ran literature .AGV and AGVS security device design [J]. Logistics Technology and Application, 2001,01: 32-34. 